\title{
A study of the Galactic star forming region IRAS 02593+6016/S 201 in infrared and radio wavelengths ${ }^{\star}, \star \star$
}

\author{
D. K. Ojha ${ }^{1,4}$, S. K. Ghosh ${ }^{1}$, V. K. Kulkarni ${ }^{2}$, L. Testi ${ }^{3}$, R. P. Verma ${ }^{1}$, and S. Vig ${ }^{1}$ \\ 1 Tata Institute of Fundamental Research, Homi Bhabha Road, Mumbai 400 005, India \\ 2 National Centre for Radio Astrophysics, Post Bag 3, Ganeshkhind, Pune 411 007, India \\ 3 Osservatorio Astrofisico di Arcetri, Largo E. Fermi 5, 50125 Firenze, Italy \\ ${ }^{4}$ National Astronomical Observatory of Japan, Osawa 2-21-1, Mitaka, Tokyo 181-8588, Japan
}

Received 11 September 2003 / Accepted 3 November 2003

\begin{abstract}
We present infrared and radio continuum observations of the S 201 star forming region. A massive star cluster is seen, which contains different classes of young stellar objects. The near-infrared colour-colour and colour-magnitude diagrams are studied to determine the nature of these sources. We have discovered knots of molecular hydrogen emission at $2.122 \mu \mathrm{m}$ in the central region of S 201. These knots are clearly seen along the diffuse emission to the north-west and are probably obscured Herbig-Haro objects. High sensitivity and high resolution radio continuum images from GMRT observations at 610 and $1280 \mathrm{MHz}$ show an arc-shaped structure due to the interaction between the HII region and the adjacent molecular cloud. The ionization front at the interface between the HII region and the molecular cloud is clearly seen comparing the radio, molecular hydrogen and Br $\gamma$ images. The emission from the carriers of Unidentified Infrared Bands in the mid-infrared 6-9 $\mu \mathrm{m}$ (possibly due to PAHs) as extracted from the Midcourse Space Experiment survey (at 8, 12, 14 and $21 \mu \mathrm{m}$ ) is compared with the radio emission. The HIRES processed IRAS maps at 12, 25, 60 and $100 \mu \mathrm{m}$ have also been used for comparison. The spatial distribution of the temperature and the optical depth of the warm dust component around the S 201 region has been generated from the mid-infrared images.
\end{abstract}

Key words. Herbig-Haro objects - stars: formation - ISM: individual objects: S 201

\section{Introduction}

The high mass star forming region S 201, corresponding to the radio source $\mathrm{W} 5 \mathrm{~A}$, is at a distance of $2.3 \mathrm{kpc}$ from the Sun (Mampaso et al. 1987). The S 201 region is coincident with an IRAS source (IRAS 02593+6016) with an increasing spectrum from 12 to $100 \mu \mathrm{m}$. The IRAS fluxes lead to a luminosity of $\sim 1.1 \times 10^{4} L_{\odot}$ (Zinchenko et al. 1997) for this source. The HII region S 201 is part of an extended complex of gas and

Send offprint requests to: D. K. Ojha, e-mail: ojha@tifr.res.in

* This paper is based on observations made with the Italian Telescopio Nazionale Galileo (TNG) operated on the island of La Palma by the Centro Galileo Galilei of the CNAA (Consorzio Nazionale per l'Astronomia e l'Astrofisica) at the Spanish Observatorio del Roque de los Muchachos of the Instituto de Astrofisica de Canarias.

$\star \star$ This publication makes use of data products from the Two Micron All Sky Survey, which is a joint project of the University of Massachusetts and the Infrared Processing and Analysis Center/California Institute of Technology, funded by the National Aeronautics and Space Administration and the National Science Foundation. IPAC is thanked for providing HIRES processed IRAS data. dust stretched in the east-west direction and is bordered to the west by the molecular cloud IC 1848 identified as the radio source W 5.

Earlier observations of S 201 in the infrared wavelengths (Kleinmann et al. 1979; Thronson et al. 1984; Mampaso et al. 1989; Carpenter et al. 1993) and the radio (Felli et al. 1987; Fich 1993; Omar et al. 2002) have shown an interesting structure presumably due to the interaction between the HII region and the adjacent molecular cloud (Martin \& Barrett 1978). The radio source has a bright, arc-shaped edge on one side and a smoothly decreasing surface brightness distribution on the opposite side as seen in the VLA observations at 2 and $6 \mathrm{~cm}$ (Felli et al. 1987). Felli et al. (1987) modeled this configuration in terms of a three-dimensional electron distribution produced by the ionizing radiation of an early-type star located outside a spherical molecular cloud and found that an O9 zero age main sequence (ZAMS) star placed at a distance of $0.38 \mathrm{pc}$ from a molecular cloud of $0.42 \mathrm{pc}$ radius allows a good fit to the their data.

We have started a near-infrared (NIR) and radio continuum observational programme of regions of massive star formation. In this paper we study the Galactic star forming region S 201 

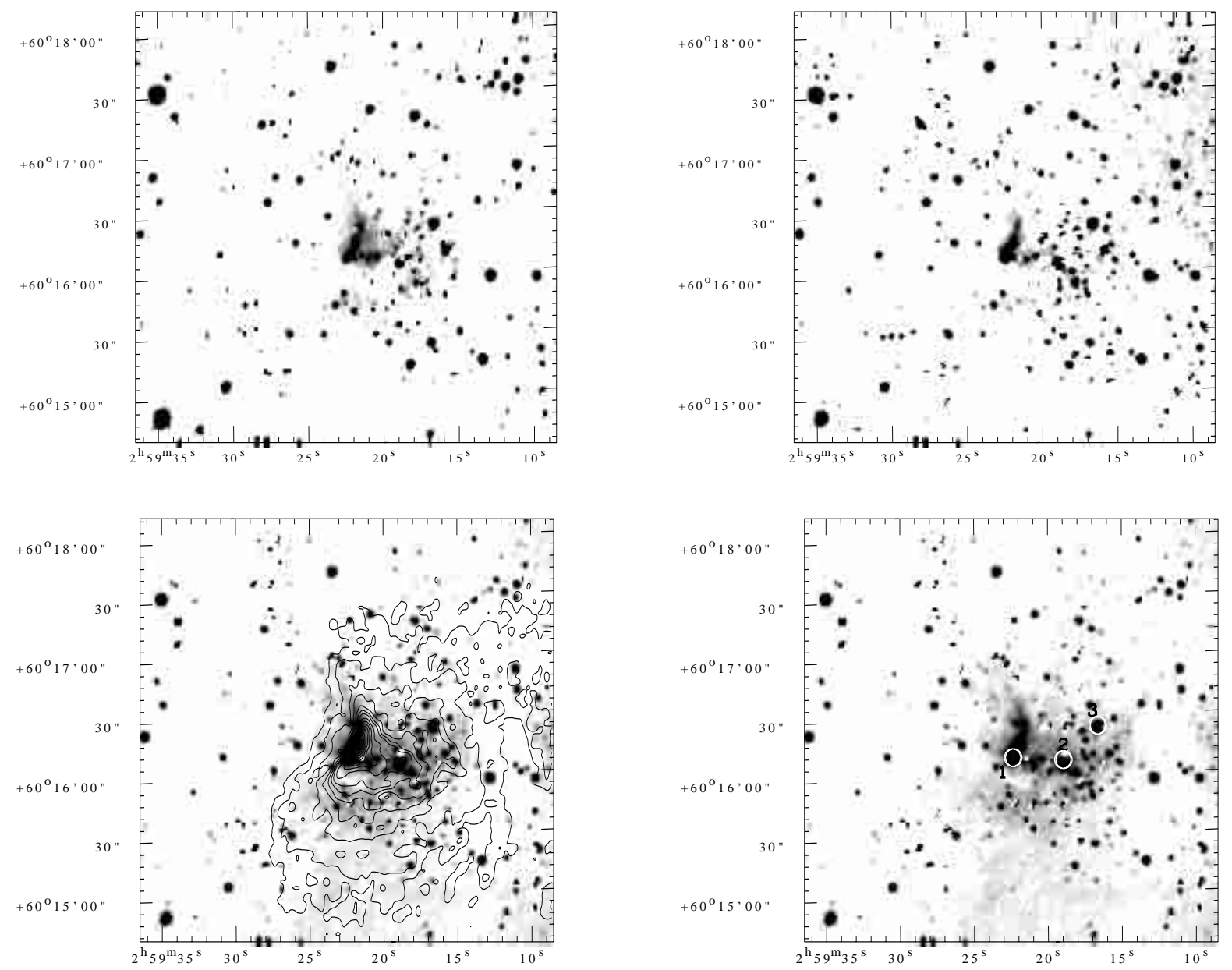

Fig. 1. TIRGO $J, H$ and $K$ band images (clockwise from the top left) of S 201 region. North is up and east to the left. The total integration times are $60 \mathrm{~s}$ in $J, 24 \mathrm{~s}$ in $H$, and $24 \mathrm{~s}$ in $K$ bands. The abscissa and the ordinates are in B1950.0 epoch. The bottom left figure shows the TIRGO $K$ band image overlayed by GMRT radio contours (see Sect. 3.2 for discussion).

in the infrared and radio wavelengths with the aim of identifying the stellar populations in the directions of the IRAS source (IRAS 02593+6016). This paper combines new NIR observations from the Telescopio Infrarosso del Gornergratm (TIRGO) and Telescopio Nazionale Galileo (TNG) telescopes, Italy with mid-infrared (MIR) data $(8-21 \mu \mathrm{m})$ from the Midcourse Space Experiment (MSX) and radio continuum observations at 610 and $1280 \mathrm{MHz}$ from the Giant Metrewave Radio Telescope (GMRT), India.

In Sect. 2, we present broad- and narrow-band NIR observations, as well as complementary MIR and radio continuum observations. Section 3 deals with the results and the discussion and we summarize our conclusions in Sect. 4.

\section{Observations and data reduction}

\subsection{NIR observations}

\subsubsection{Broad-band images}

The broad-band $(J H K)$ NIR observations were carried out on 23 December 2000 using the Arcetri NIR camera (ARNICA) mounted on the $1.5 \mathrm{~m} \mathrm{f} / 20$ infrared telescope TIRGO. ARNICA is equipped with a NICMOS3 $256 \times 256 \mathrm{HgCdTe}$ detector. The plate scale at TIRGO was $0.96^{\prime \prime} /$ pixel and the mean PSF was approximately $1.5^{\prime \prime}-1.8^{\prime \prime}(F W H M)$ during the observations.

We observed the field in the direction of the IRAS source $02593+6016$ in the three standard $J(1.25 \mu \mathrm{m}, \Delta \lambda=0.3 \mu \mathrm{m})$, $H(1.6 \mu \mathrm{m}, \Delta \lambda=0.3 \mu \mathrm{m})$, and $K(2.2 \mu \mathrm{m}, \Delta \lambda=0.4 \mu \mathrm{m})$ broad-band filters. A large number of dithered sky frames were obtained (by shifting the telescope a few arcmin off the source in north-west-south-east directions) in all the filters for sky subtraction and for making flat frames. Total on-target integration times were $60 \mathrm{~s}, 24 \mathrm{~s}$ and $24 \mathrm{~s}$ in the $J, H$ and $K$ bands, respectively. The photometric calibration was obtained by observing the standard stars AS03 and AS09 (Hunt et al. 1998) in all three bands. Figure 1 shows the $J, H$, and $K$ band images of S 201 region. The $J H K$ images show the presence of diffuse emission near the center of the images, with an apparent concentration of embedded stars, suggesting the presence of a young cluster (see Sect. 3).

Data reduction was done using IRAF $^{1}$ software tasks. All the NIR images went through standard pipeline procedures

${ }^{1}$ IRAF is distributed by the National Optical Astronomy Observatories, which is operated by the Association of Universities for Research in Astronomy, Inc. under contract to the National Science Foundation. 

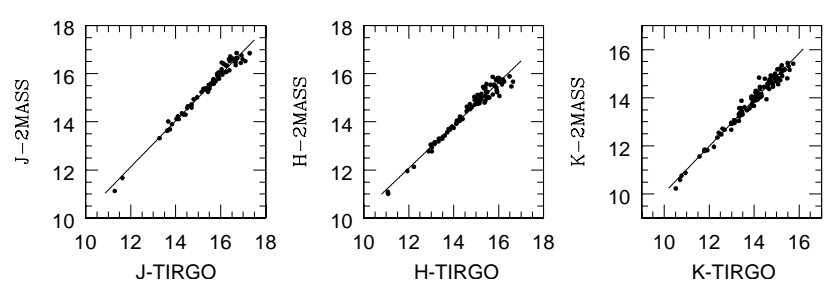

Fig. 2. Comparison between TIRGO and 2MASS magnitudes in $J, H$ and $K$ bands. The continuous lines show the linear fit to the magnitudes. The slopes of the linear fits range from 0.9 to 0.97 between the two systems in the three filters.

such as sky-subtraction and flat-fielding. Accurate photometry was performed on the point sources detected in each of the three bands using the DAOPHOT (Stetson 1987) routines in IRAF software. Absolute position calibration was achieved using the coordinates of a number of stars from the USNO2.0 catalogue. The completeness limits of the images were evaluated by adding artificial stars of different magnitudes to the images and determining the fraction of stars recovered in each magnitude bin. The recovery rate was greater than $90 \%$ for magnitudes brighter than 17, 16 and 15.5 in the $J, H$ and $K$ bands, respectively. The observations are complete (100\%) to the level of $15.5,14.5$ and 14 mag in $J, H$ and $K$ bands, respectively. The average photometric error in all colours is $\pm 0.07 \mathrm{mag}$.

We checked our photometry with the data from 2MASS (The Two Micron All Sky Survey). The 2MASS All Sky Point Source Catalogue ${ }^{2}$ provides $J, H$ and $K_{\mathrm{s}}$ magnitudes for each source. The 2MASS $K_{\mathrm{s}}$ filter is centered at $2.17 \mu \mathrm{m}$ and has a bandpass of $0.32 \mu \mathrm{m}$. Figure 2 shows the comparison between TIRGO and 2MASS magnitudes in $J, H$ and $K$ filters. We see a good linear relation between the two systems, with the slopes between 0.9-0.97 and dispersions that increase with magnitude.

\subsubsection{Narrow-band images}

The images through narrow-band filters including the molecular hydrogen $\left(\mathrm{H}_{2} v=1-0 \mathrm{~S}(1)\right)$ transition $(2.122 \mu \mathrm{m}, F W H M=$ $0.032 \mu \mathrm{m})$, the $\operatorname{Br} \gamma$ line $(2.169 \mu \mathrm{m}, F W H M=0.035 \mu \mathrm{m})$, and continuum $\mathrm{K}$ (Kcont at $2.275 \mu \mathrm{m}, F W H M=0.039 \mu \mathrm{m}$ ), were obtained on 13 November, 2002 with the $3.58 \mathrm{~m}$ TNG telescope at La Palma using the Near-Infrared Camera Spectrometer (NICS). NICS is the TNG infrared $(0.9-2.5 \mu \mathrm{m})$ multimode instrument based on a HgCdTe Hawaii $1024 \times 1024$ array (Baffa et al. 2001). The image scale was $0.25^{\prime \prime} /$ pixel and the mean PSF was approximately $1.3^{\prime \prime}(F W H M)$. The total integration time was $90 \mathrm{~s}$ in all three filters. The images were analysed in a similar process as those from TIRGO. To identify the pure line emission $\left(\mathrm{H}_{2} v=1-0 S(1)\right.$ and $\left.\mathrm{Br} \gamma\right)$ ) one needs to subtract the continuum. This is done by subtracting the $K$ continuum image from $\mathrm{H}_{2}$ and $\mathrm{Br} \gamma$ images after aligning and PSF matching.

Figure 3 shows the $\mathrm{H}_{2}, \mathrm{Br} \gamma, \mathrm{H}_{2}$ continuum subtracted (revealing the pure $\mathrm{H}_{2}$ line emission) and $\mathrm{Br} \gamma$ continuum subtracted images.

\footnotetext{
${ }^{2}$ http://www.ipac.caltech.edu/cgi-bin/gator/nph-dd
}

\subsection{Mid-infrared data from MSX}

The MSX images in $A(8.3 \mu \mathrm{m}), C(12.13 \mu \mathrm{m}), D(14.65 \mu \mathrm{m})$ and $E(21.34 \mu \mathrm{m})$ bands (Price et al. 2001) for the region around S 201 have been used to estimate the spatial distribution of warm interstellar dust, its temperature and optical depth. The MSX $A$ and $C$ bands with $\lambda(\Delta \lambda)$ corresponding to $8.28(3.36)$ and 12.13(1.72) include several Unidentified Infrared emission Bands (UIBs) at 6.2, 7.7, 8.7, 11.3, and $12.7 \mu \mathrm{m}$. Using a scheme developed by Ghosh \& Ojha (2002), the emission in these UIBs, probably due to Polycyclic Aromatic Hydrocarbons (PAHs), have been extracted from the MSX images by correcting for the underlying thermal continuum from the interstellar dust.

\subsection{Mid- and far-infrared data from IRAS}

The data from the IRAS survey in the four bands $(12,25,60$ and $100 \mu \mathrm{m}$ ) for the region around S 201 were HIRES processed (Aumann et al. 1990) at IPAC. The HIRES processed maps in all the four IRAS bands are shown in Fig. 4. These maps have also been used to generate the maps of dust colour temperature and optical depth.

\subsection{GMRT Radio continuum observations}

The ionized gas within and around the HII region associated with S 201 has been mapped at high angular resolution using the Giant Metrewave Radio Telescope (GMRT) array. The radio continuum observations were carried out in two frequency bands, 610 and $1280 \mathrm{MHz}$ on 4 January, 2002 and 28 September, 2002, respectively. The sources 3C 48 and 3C 147 were used as the primary flux calibrators for the 610 and $1280 \mathrm{MHz}$ observations, respectively, while the source $0432+416$ was used as a secondary calibrator for both the observations. The GMRT antennas and their configurations are discussed in detail by Swarup et al. (1991).

Data reduction was done in classic AIPS. Bad data (dead antennas, interference, spikes, etc.) were identified and flagged using UVFLG and TVFLG. Images of the field were formed by Fourier inversion and cleaning (IMAGR). The initial images were improved by self-calibration (CALIB) in both phase and amplitude.

Figures 5 and 6 show the radio continuum images of S 201 generated from the GMRT observations at 1280 and $610 \mathrm{MHz}$ respectively. The radio continuum images have a resolution of $4.5^{\prime \prime} \times 2.5^{\prime \prime}$ at $1280 \mathrm{MHz}$ and $8.7^{\prime \prime} \times 5.7^{\prime \prime}$ at $610 \mathrm{MHz}$. The images have an rms of $42 \mu \mathrm{Jy}^{\text {beam }}{ }^{-1}$ at $1280 \mathrm{MHz}$ and $47 \mu \mathrm{Jy} \mathrm{beam}^{-1}$ at $610 \mathrm{MHz}$. The total flux densities are $0.68 \mathrm{Jy}$ and $0.77 \mathrm{Jy}$ at $1280 \mathrm{MHz}$ and $610 \mathrm{MHz}$, respectively.

\section{Results and discussion}

\subsection{The embedded cluster}

Inside a $\sim 3.5^{\prime} \times 3.4^{\prime}$ field centered on the IRAS $02593+6016$ source, 114 stars were found to be common to all the three $J H K$ bands and 153 stars common to the $H K$ bands alone, with 

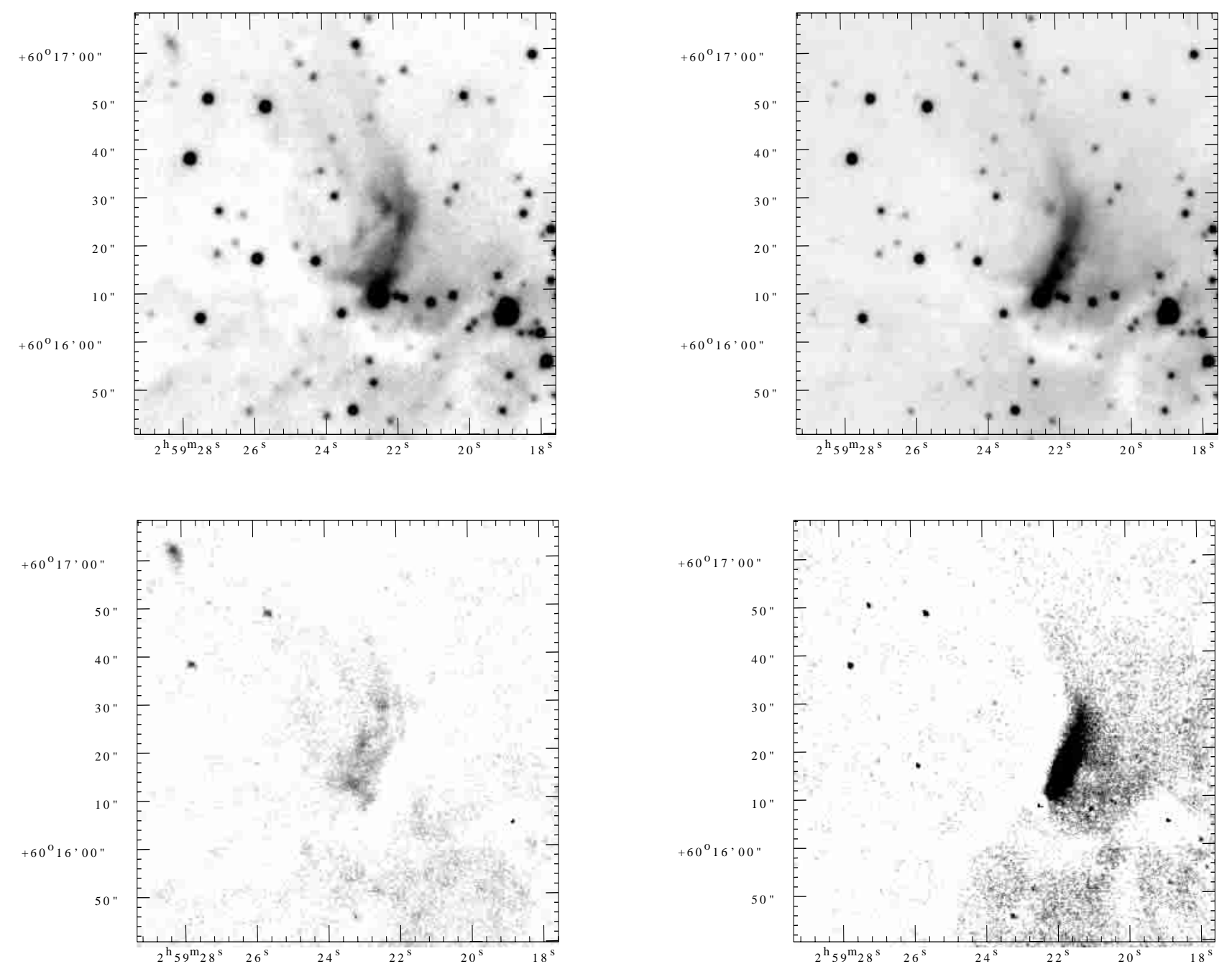

Fig. 3. $\mathrm{H}_{2}, \mathrm{Br} \gamma$, continuum subtracted $\mathrm{Br} \gamma$, and continuum subtracted $\mathrm{H}_{2}$ line images of the central region $\left(\sim 88^{\prime \prime} \times 88^{\prime \prime}\right)$ of $\mathrm{S} 201$ (clockwise from the top left). Notice also the presence of $\mathrm{H}_{2}$ knots near the center and upper left corner of the image (bottom left figure). North is the top and east to the left. The total integration time is $90 \mathrm{~s}$ in all the filters. The abscissa and the ordinates are in B1950.0 epoch.

magnitude errors less than 0.2. Figure 7 shows a colour-colour (CC) diagram for the 114 stars detected in the $J H K$ bands. The solid and broken heavy curves represent the unreddened main sequence dwarfs and giant branch (Koornneef 1983) and the parallel dashed lines are the reddening vectors for early and late type stars (drawn from the base and tip of the two branches) that encloses reddened main sequence objects. The dotted line indicates the locus of T-Tauri stars (Meyer et al. 1997). We have assumed that $A_{J} / A_{V}=0.282 ; A_{H} / A_{V}=0.175$ and $A_{K_{\mathrm{s}}} / A_{V}=0.112$ (Rieke \& Lebofsky 1985).

Most of the sources have colours of reddened photospheres but some of the stars lying outside the region of reddened main sequence objects (right of the reddening line for early type stars) are mostly young stellar objects (YSOs) with intrinsic colour excess. By de-reddening the stars (on the CC diagram) that fall within the reddening vectors encompassing the main sequence stars, we found the visual extinction $\left(A_{V}\right)$ towards each star. The individual extinction values range from 0 to $18 \mathrm{mag}$ with an average foreground extinction of $A_{V} \sim 5 \mathrm{mag}$. The stars lying on the left side of the reddening band are mostly foreground stars as supported by their low values of $A_{V}$.
About $12 \%$ of the sources (within $0.43 \mathrm{pc}$ radius around the position of the IRAS source) detected in $J, H$ and $K$ bands show an infrared excess $\left(H-K>1, J-H>1, A_{V} \simeq 8-14\right)$. These YSOs are shown as star symbols in Fig. 7 and they are concentrated close to the center of the embedded young stellar cluster. The most massive O6-O8 type star is located S-W of the cluster and is surrounded by at least two massive stars of spectral types earlier than B2, both of them showing an infrared excess (see Fig. 8).

Figure 8 shows the $H-K$ vs. $K$ colour-magnitude (CM) diagram for all the sources detected in $H K$ bands. The vertical solid lines (from the left to the right) represent the main sequence curve reddened by 0,20 and $40 \mathrm{mag}$, respectively. We have assumed a distance of $2.3 \mathrm{kpc}$ to the source to reproduce the main sequence data on this plot. The horizontal slanting lines in Fig. 8 trace the reddening zones for each spectral type. However, it should be noted that the spectral types inferred from the CM diagram are only upper limits when the stars present infrared excess. YSOs with an infrared excess found from CC diagram (Fig. 7) are shown as star symbols. However, it is important to note that even those stars not shown with a 

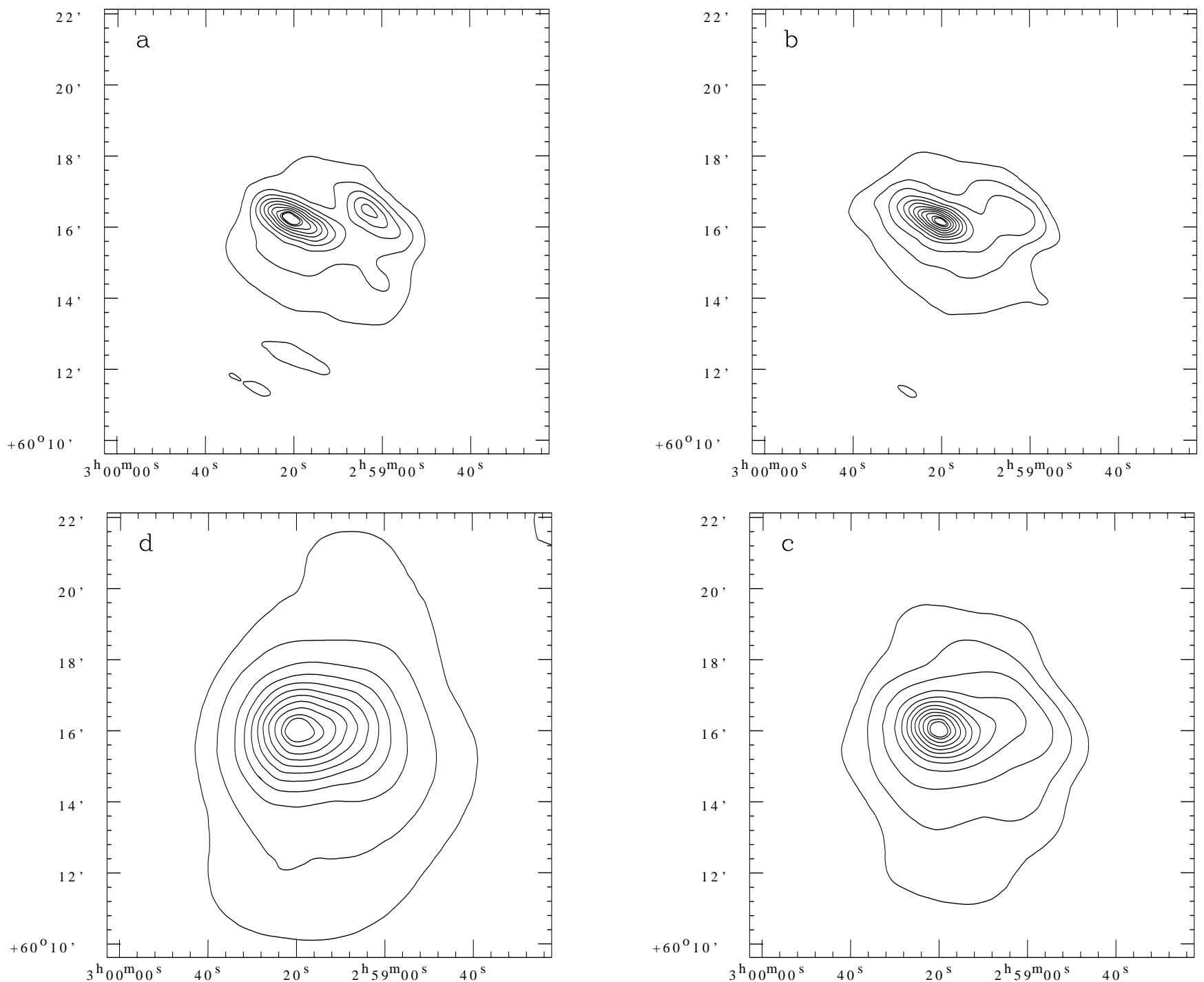

Fig. 4. The HIRES processed IRAS maps for S 201 in the four bands (clockwise from the top left) - a) $12 \mu \mathrm{m}$ with peak $=21 \mathrm{Jy} / \mathrm{sq}$ arcmin, b) $25 \mu \mathrm{m}$ with peak $=122 \mathrm{Jy} / \mathrm{sq}$ arcmin, c) $60 \mu \mathrm{m}$ with peak $=622 \mathrm{Jy} / \mathrm{sq}$ arcmin, and d) $100 \mu \mathrm{m}$ with peak $=415 \mathrm{Jy} / \mathrm{sq}$ arcmin. The isophot contour levels in $12 \mu \mathrm{m}$ are 95, 90, 80, 70, 60, 50, 40,30, 20 and 10\%, in 25 and $60 \mu \mathrm{m}$ are 95, 90, 80, 70, 60, 50, 40, 30, 20, 10, 5 and 2.5\%, and in $100 \mu \mathrm{m}$ are 95, 90, 80, 70, 60, 50, 40,30, 20, 10 and 5\% of the respective peaks. The abscissa and the ordinates are in B1950.0 epoch.

star symbol may represent YSOs with intrinsic colour excess, since these stars detected in the $H$ and $K$ bands are not detected in the $J$ band due to their very red colours. The three massive and luminous stars discussed earlier are located at the top of the CM diagram $(K<11.8)$.

Following the method described in Testi et al. (1999) we estimated the number of stars in the central regions of the cluster, within $40^{\prime \prime}(0.4 \mathrm{pc})$ of the most massive star in the cluster (\#2). We found an excess of $\sim 38$ objects above the background with $K$ band magnitude less than 15.5 , corresponding to our $90 \%$ completeness level. The corresponding stellar volume density in the inner region of the cluster would then be $\sim 10^{2} \mathrm{stars} / \mathrm{pc}^{3}$, on the low side compared to the typical densities of objects around early Herbig Be stars in the survey of Testi et al. (1999). However, to compare we need to estimate the lowest mass object that can be probed with our observations, which can be estimated following the method outlined in Testi et al. (1998). Assuming the distance of $2.3 \mathrm{kpc}$, an age in the range $0.5-1 \mathrm{Myr}$, and an extinction in $K$ band between 0 and 1 mag (up to $A_{V} \sim 10$ ), the derived magnitude limit corresponds to $M \sim 0.5 M_{\odot}$ (using the Palla \& Stahler 1999 pre-main sequence evolutionary tracks), which is significantly higher than most of the limits in Testi et al. (1999).

\subsection{Comparison of infrared and radio continuum observations}

The radio maps from GMRT at 1280 and $610 \mathrm{MHz}$ (Figs. 5 and 6) display a striking cometary morphology with sharp boundaries toward the N-NE, and diffuse emission extending toward S-SW (an angular diameter of approximately $\sim 4^{\prime}$ ). This kind of morphology is produced by the winds and shocks from the massive stars in the vicinity of molecular clouds, which are expected to compress the nearmost edges of nearby clouds and develop the cometary structure (Bachiller et al. 2002). Three bright near-infrared sources are present within the radio 


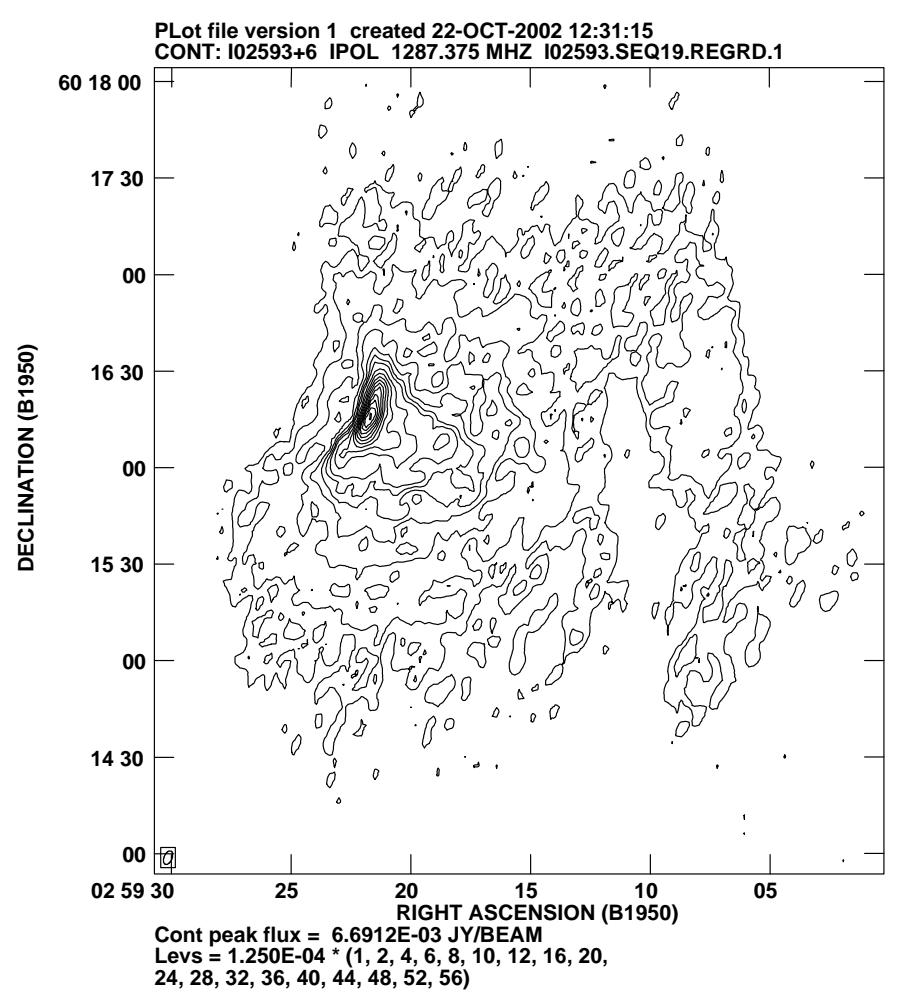

Fig. 5. GMRT high resolution map of $\mathrm{S} 201$ at $1280 \mathrm{MHz}$. The resolution is $4.5 \times 2.5 \operatorname{arcsec}^{2}$ along $\mathrm{PA}=-23^{\circ}$, and the rms noise in the map is $42 \mu \mathrm{Jy}_{\text {beam }}{ }^{-1}$.

nebulosity. One of the sources (id\#1 in Fig. 1) with NIR excess $(H-K>1)$ is very close to the radio peak suggesting the spectral type of B1 in the $H-K$ vs. $K$ CM diagram. This source is also coincident, within errors, with the $\mathrm{H}_{2} \mathrm{O}$ maser position present in the region (Blair et al. 1980). The brightest infrared source (id\#2; $K=10.24, H-K=1.40, J-H=2.66$ ) deeply embedded in the cloud $\left(A_{V} \sim 22\right)$, is located $\sim 26^{\prime \prime}(\sim 0.29 \mathrm{pc})$ west of the radio peak. The projected distance of source \#2 from the cloud is approximately $33^{\prime \prime}(0.37 \mathrm{pc})$. The colour and IR luminosity of this source indicate that it is an O6-O8 type star. The position of \#2 coincides with the "secondary peak" detected by Felli et al. (1987) in the radio and also seen in the GMRT radio images (see Figs. 1 and 5).

It is interesting to revisit the model of Felli et al. (1987) after the results of our new near-infrared observations. Their modeling of the VLA observations was based on the assumption of a single star providing all the ionizing photons, while our observations clearly reveal a rich cluster within the radio continuum emission. The estimated spectral types from the CM diagram and the total Lyman continuum photons (using the Table in Panagia 1973) for the five bright stars within the young cluster are shown in Table 1. The most massive star (\#2) alone provides more than $90 \%$ of the total Lyman continuum supply rate, explaining the success of the model based on a single source of the ionizing radiation. It is also interesting to note that our observations confirm the suggestion by Felli et al. (1987) of the co-location of the massive star responsible for the ionization of the nebula and the secondary peak of radio emission.

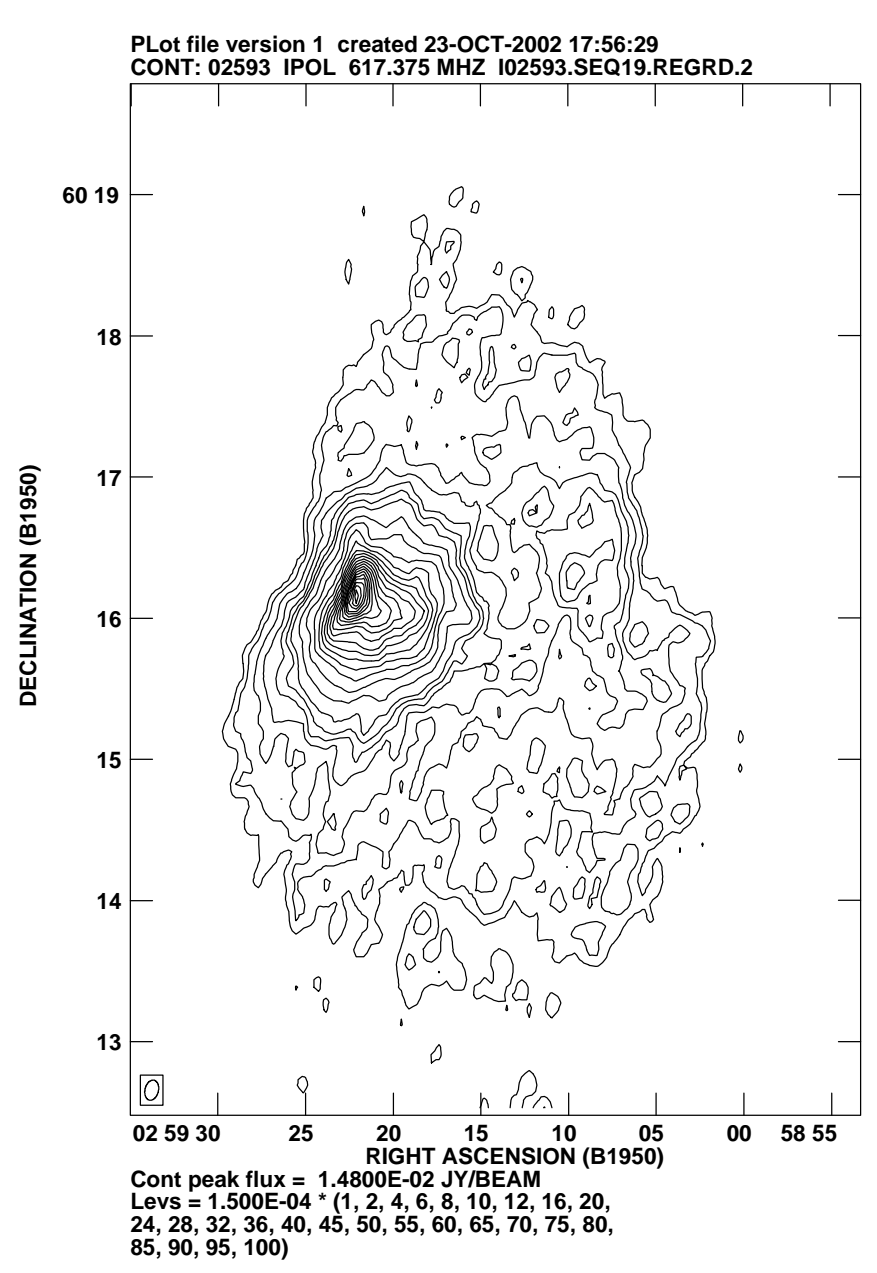

Fig. 6. GMRT high resolution map of S 201 at $610 \mathrm{MHz}$. The resolution is $8.7 \times 5.7 \operatorname{arcsec}^{2}$ along PA $=-14^{\circ}$, and the rms noise in the map is $47 \mu \mathrm{Jy}_{\text {beam }}{ }^{-1}$.

The quadrupole transition $(v=1-0 S(1))$ of molecular hydrogen at $2.122 \mu \mathrm{m}$ is an excellent tracer of shock emission and photo-dissociation regions (PDRs). In particular, in PDRs the molecular hydrogen emission traces the first neutral layer beyond the ionization front; a typical, well studied case is that of the PDR at the interface between the Orion Nebula and the Orion molecular cloud (the Orion Bar, see Walmsley et al. 2000). The model of Felli et al. (1987) implicitly predicts that a PDR should be located at the interface between the ionized and molecular gas, to the east of the arc-shaped feature in the radio continuum maps. Our near-infrared narrow band images fully support this picture, as we detect diffuse $\mathrm{H}_{2}$ emission where the PDR region should be located (see Fig. 9). Additionally, in the north-east corner of the image we detect an $\mathrm{H}_{2}$ knot reminiscent of Herbig-Haro objects.

The continuum-subtracted $\mathrm{Br} \gamma$ image displays a morphology very similar to the radio continuum maps, with a bright, arc-shaped edge on one side and a smoothly decreasing surface brightness distribution on the opposite side and a peak coinciding exactly with the radio continuum one (Figs. 3 and 10). The similar morphology between the $\mathrm{Br} \gamma$ and radio continuum maps indicates that there are no steep extinction gradients across S 201. 


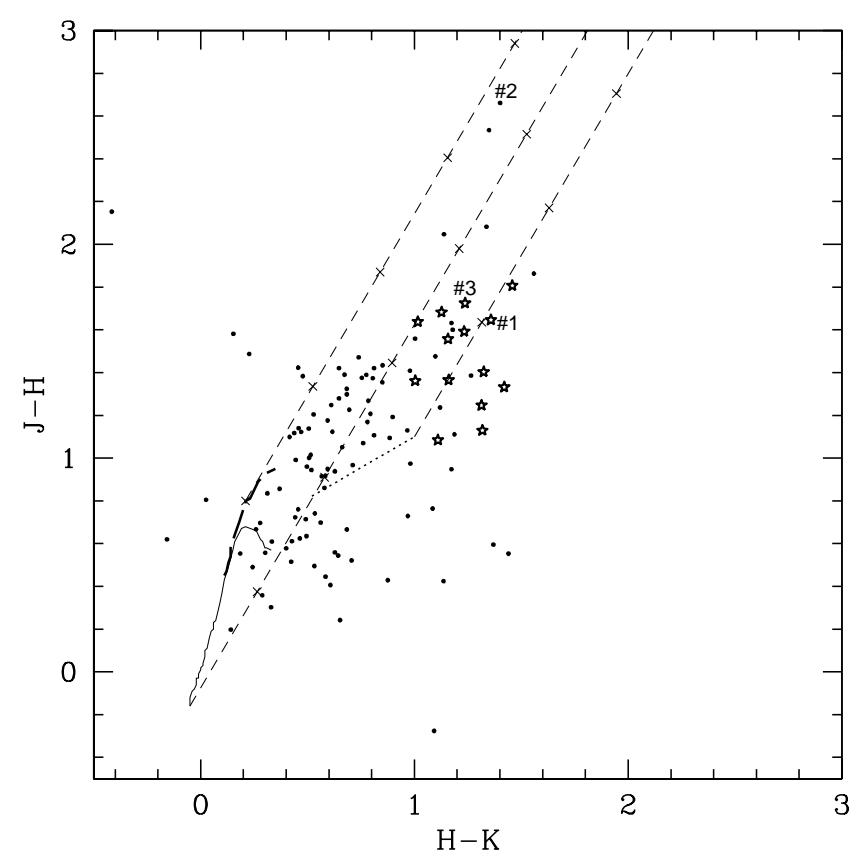

Fig. 7. Colour-colour diagram for the 114 sources detected in $J H K$ bands in S 201 region. The locii of the main sequence and giants branch are shown by the solid curve (dwarf) and broken heavy curve (giants) taken from Koornneef (1983). The three parallel dashed straight lines follow the reddening vectors taken from Rieke \& Lebofsky (1985). Crosses on the dashed lines are separated by $A_{V}=5 \mathrm{mag}$. The dotted line represents the locus of T-Tauri stars (Meyer et al. 1997). The star symbols represent YSOs with intrinsic colour excesses within $0.43 \mathrm{pc}$ radius around the center of the cluster. Three bright stars within the cluster are represented by \#1, \#2 and \#3 (see Fig. 1 and Sect. 3).

The ionization front at the interface between the HII region and the molecular cloud is clearly seen by comparing the radio, molecular hydrogen and $\mathrm{Br} \gamma$ images (Figs. 3, 5 and 6). A more detailed analysis from the GMRT radio observations will be presented in a future paper.

\subsection{Spatial distribution of Unidentified Infrared emission Bands (UIBs) around S 201 region}

The MSX surveyed the entire Galactic plane within $|b| \leq 5^{\circ}$ in four mid-infrared spectral bands centered at 8.28, 12.13, 14.65 and $21.34 \mu \mathrm{m}$, with image resolution of $20^{\prime \prime}$ (Price et al. 2001). We have used the scheme developed by Ghosh \& Ojha (2002) to extract the contribution of Unidentified Infrared emission Bands (UIBs) from the mid-infrared MSX images of S 201 region in the four bands. The scheme models the observations with a combination of thermal emission (gray body) from interstellar dust and the UIB emission from the gas component, under reasonable assumptions. The spatial distribution of emission in the UIBs with an angular resolution $\sim 20^{\prime \prime}$ (intrinsic to MSX survey) was extracted and is shown in Fig. 11.

The UIB emission map is compared with the GMRT observations at $1280 \mathrm{MHz}$ in Fig. 12. The positions of the peaks

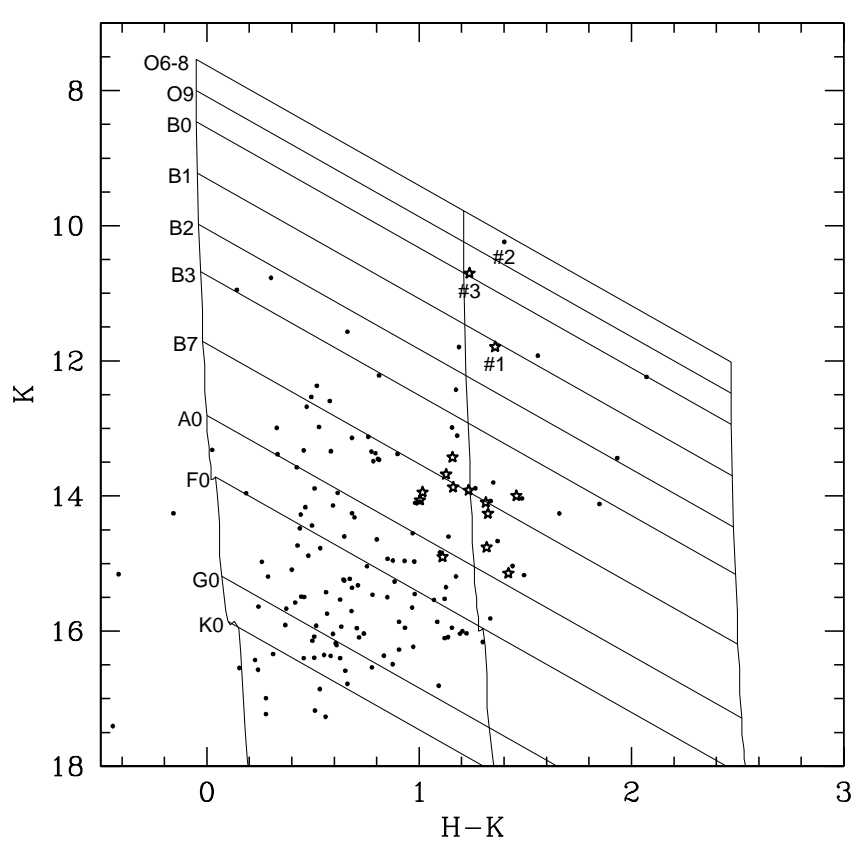

Fig. 8. Colour-magnitude diagram for the 153 sources detected in $H K$ bands. Stars represent the YSOs identified from Fig. 7. The vertical solid lines from left to right indicate the track of main sequence dwarfs at $2.3 \mathrm{kpc}$ reddened by 0,20 and $40 \mathrm{mag}$, respectively. The intrinsic colours are taken from Koornneef (1983). Slanting horizontal lines identify the reddening vectors (Rieke \& Lebofsky 1985). The symbols are same as shown in Fig. 7.

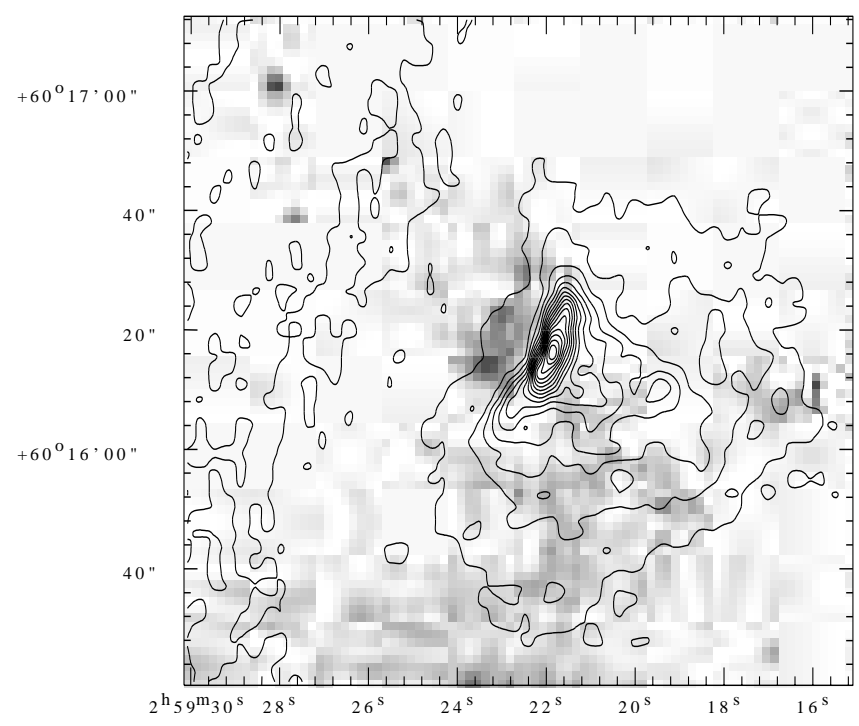

Fig. 9. Continuum-subtracted molecular hydrogen emission at $2.122 \mu \mathrm{m}$ around S 201 region. The contour plot shows the $1280 \mathrm{MHz}$ radio continuum emission from GMRT. Notice also the presence of knots near the center and upper left corner of the image. The abscissa and the ordinates are in B1950.0 epoch.

and other morphology (along N-S to E-W) compare rather well between the UIBs and the radio emission maps, although we see a strong extended component of UIB emission along S-E as compared to the radio emission. 
Table 1. Bright stars within the young cluster.

\begin{tabular}{cccccccc}
\hline \hline $\begin{array}{c}\text { RA (B1950) } \\
\text { hh:mm:ss }\end{array}$ & $\begin{array}{c}\text { Dec (B1950) } \\
\text { dd:mm:ss }\end{array}$ & $\begin{array}{c}J \\
\mathrm{mag}\end{array}$ & $\begin{array}{c}H \\
\mathrm{mag}\end{array}$ & $\begin{array}{c}K \\
\mathrm{mag}\end{array}$ & $\begin{array}{c}\text { Sp. type } \\
\text { (from CM) }\end{array}$ & $\begin{array}{c}\text { Lyman continuum photons }{ }^{1} \\
\text { (Photons/s) }\end{array}$ & $\begin{array}{c}\text { Id. } \\
\text { (Figs. 1, 7 and 8) }\end{array}$ \\
\hline 02:55:17.72 & $+60: 04: 01.2$ & 13.28 & 12.23 & 11.57 & B2.5 & $2.5 \times 10^{44}$ & \\
$02: 55: 21.45$ & $+60: 04: 27.8$ & 13.67 & 11.94 & 10.70 & B0 & $2.3 \times 10^{47}$ & $\# 3$ \\
$02: 55: 22.65$ & $+60: 03: 59.2$ & 16.17 & 14.14 & 12.99 & B4 & - & \\
$02: 55: 23.74$ & $+60: 04: 09.2$ & 14.30 & 11.64 & 10.24 & O6-O8 & $5.7 \times 10^{48}$ & $\# 2$ \\
$02: 55: 27.28$ & $+60: 04: 12.6$ & 14.80 & 13.15 & 11.79 & B1 & $1.9 \times 10^{45}$ & $\# 1$ \\
\hline
\end{tabular}

${ }^{1}$ From Panagia (1973).

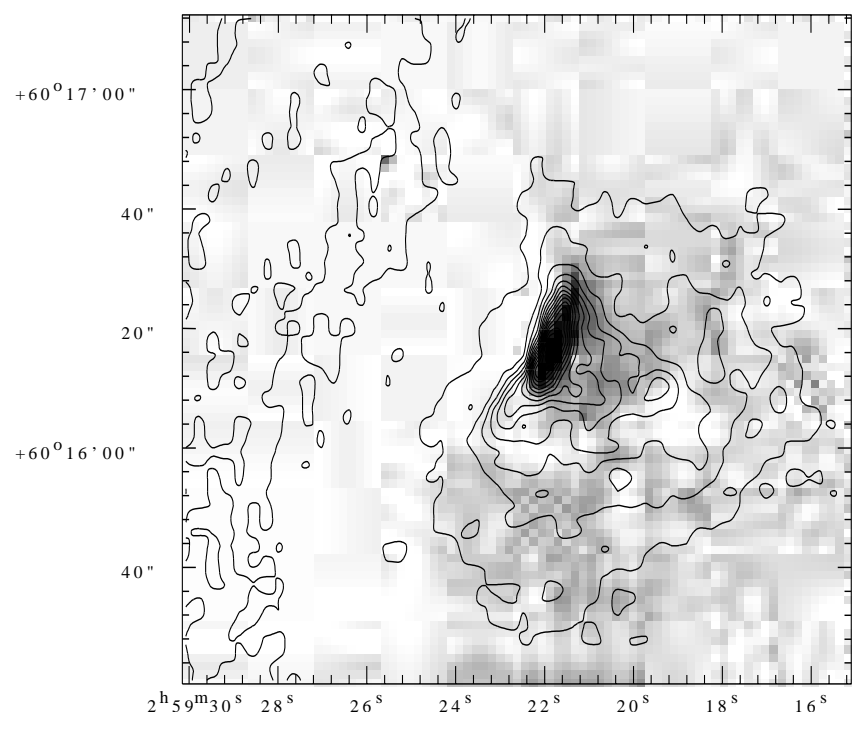

Fig. 10. Continuum-subtracted $\mathrm{Br} \gamma$ image of S 201 region. The contour plot shows the $1280 \mathrm{MHz}$ radio continuum emission from GMRT. The abscissa and the ordinates are in B1950.0 epoch.

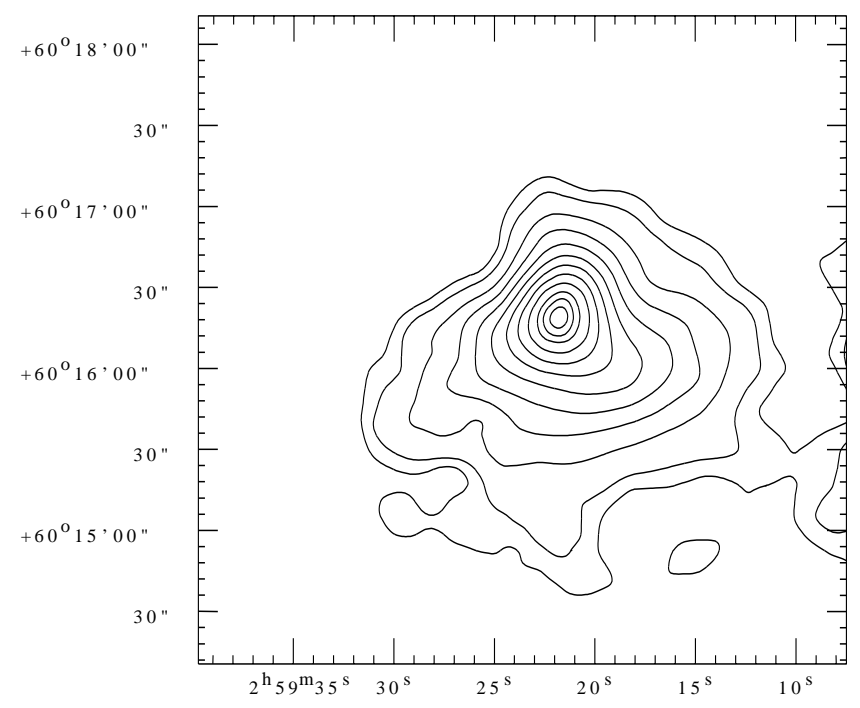

Fig. 11. The spatial distribution of total radiation in Unidentified Infrared emission Bands (UIBs) for the region around S 201, as extracted from the MSX images. Contour levels are drawn at 5, 10, $20,30,40,50,60,70,80,90,95$ and $99 \%$ of the peak intensity of $4.11 \times 10^{-5} \mathrm{Wm}^{-2} \mathrm{sr}^{-1}$. The abscissa and the ordinates are in B1950.0 epoch.

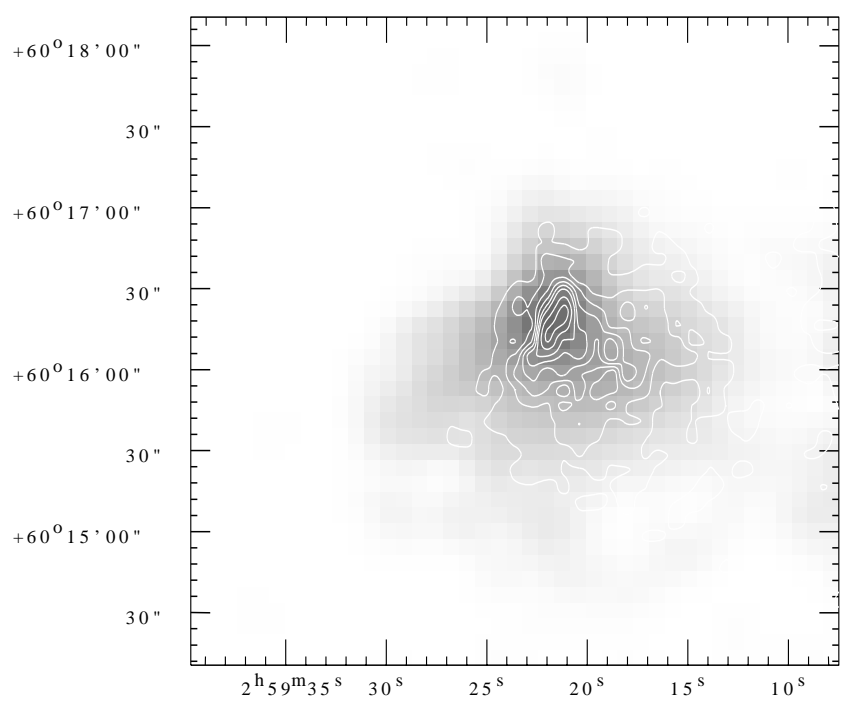

Fig. 12. The emission in UIBs (grey scale) in the mid-infrared $(6-9 \mu \mathrm{m})$. The contours show the radio emission at $1280 \mathrm{MHz}$ as measured by GMRT. The abscissa and the ordinates are in B1950.0 epoch.

\subsection{Dust optical depth and temperature maps}

\subsubsection{From MSX}

The MSX maps were used to obtain maps of warm dust temperature and optical depth (Ghosh \& Ojha 2002). Since the range of frequencies covered by the MSX bands is limited, we assume a power law dependence of the dust emissivity on frequency of the form $\epsilon_{\lambda} \propto \lambda^{-1}$. The optical depth and dust temperature maps are presented in Fig. 13.

The positions of the peaks of the optical depth and temperature are different. The optical depth map is morphologically similar to the UIB intensity map and the peaks in the two coincide indicating presence of high densities near the embedded sources. However, the temperature map shows a more extended distribution with a decreasing temperature gradient towards the $\mathrm{S}$ to $\mathrm{S}-\mathrm{W}$. The temperature map peaks close to the boundary of UIB emission toward the west $\left(48^{\prime \prime}\right.$ or $0.5 \mathrm{pc}$ from the UIBs emission peak). 

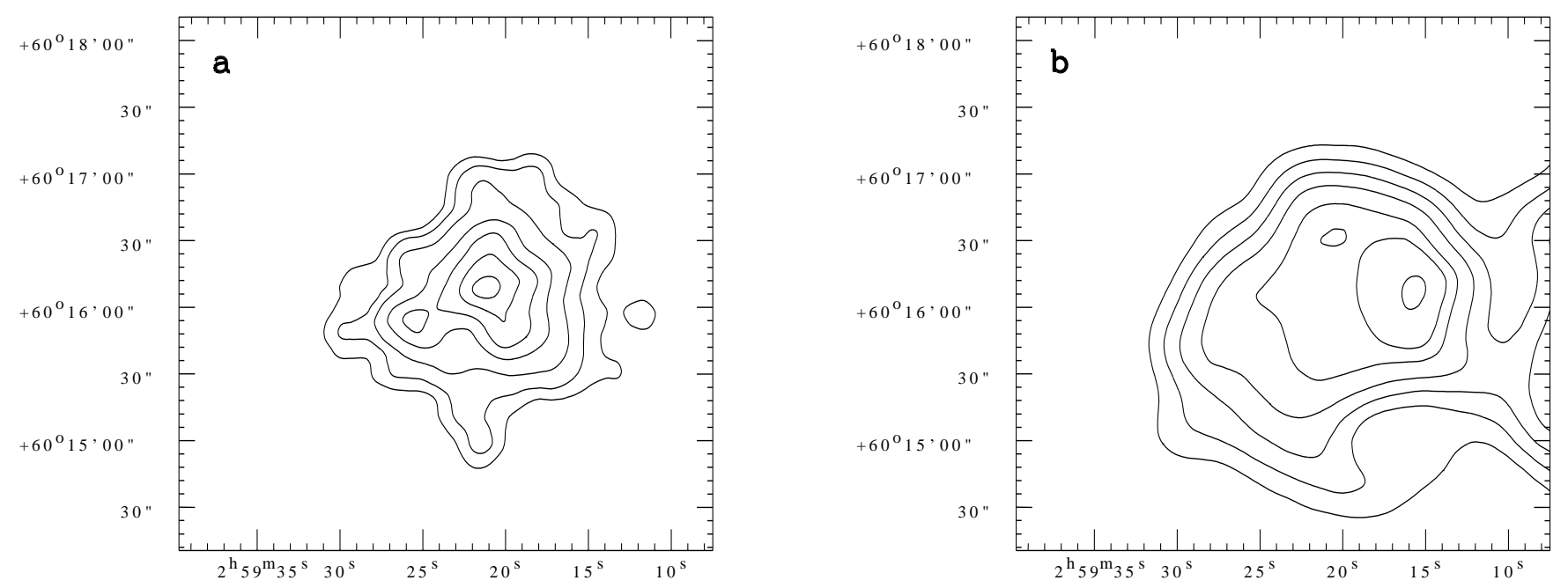

Fig. 13. a) The spatial distribution of dust optical depth $\left(\tau_{10}\right.$ at $\left.10 \mu \mathrm{m}\right)$ for the $\mathrm{S} 201$ region, as extracted from the MSX images. The contour levels are at $\tau_{10}=0.1 \times 10^{-4}, 0.2 \times 10^{-4}, 0.4 \times 10^{-4}, 0.8 \times 10^{-4}, 1.2 \times 10^{-4}, 1.6 \times 10^{-4}$ and $2.0 \times 10^{-4}$. The peak value in this map is $2.13 \times 10^{-4}$. b) The spatial distribution of dust temperature and the contours correspond to the temperatures $130,120,110,90,70,50$ and $30 \mathrm{~K}$. The abscissa and the ordinates are in B1950.0 epoch.

\subsubsection{From HIRES}

We have used HIRES maps to generate maps of the dust colour temperature $(T(12 / 25)$ and $T(60 / 100))$ and optical depth $\left(\tau_{12}\right.$ and $\left.\tau_{100}\right)$ around the $S 201$ region. The intensity maps at 12 , 25,60 and $100 \mu \mathrm{m}$ (Fig. 4) were spatially averaged before computing $T(12 / 25), \tau_{12}, T(60 / 100)$ and $\tau_{100}$ in a manner similar to that described by Ghosh et al. (1993) for an emissivity law of $\epsilon_{\lambda} \propto \lambda^{-1}$. The dust optical depth and temperature maps are presented in Fig. 14.

\subsubsection{Comparison between MSX and HIRES maps}

We perform a comparison of the $\tau_{10}$ maps generated from the higher angular resolution MSX maps (Fig. 13a) and that based on the IRAS HIRES maps at 12 and $25 \mu \mathrm{m}$ (the best resolution among the 4 bands). The latter is shown in Fig. 14a, which is scaled to $10 \mu \mathrm{m}$ by a $\lambda^{-1}$ emissivity law to compare with the MSX $\tau_{10}$ map. The peak optical depth and the effective $F W H M$ for the central maximum are $2.13 \times 10^{-4}$ and $54^{\prime \prime}$ respectively for the map based on MSX. The corresponding values from the IRAS-HIRES maps are $6.6 \times 10^{-5}$ and $60^{\prime \prime}$.

These derived values are in reasonable agreement considering that they are based on instruments with very different angular resolutions. The difference in the peak values of $\tau_{10}$ may be a result of two effects, viz., beam dilution and a clumpy interstellar medium.

The peak dust column density implied by the optical depth map at $100 \mu \mathrm{m}$ (Fig. 14b) is much higher than that implied by the $\tau_{10}$ map from MSX data (Fig. 13a). This is not surprizing since the former has its main contribution from the cooler component of dust grains $(T<80 \mathrm{~K})$ lying in the larger outer envelope of the cloud. Only the dust grains very close to the exciting source can contribute to the $\tau_{10}$ map.

Compared to MSX, the HIRES maps at 60 and $100 \mu \mathrm{m}$ trace the distribution of colder dust $(35-55 \mathrm{~K})$. However, the hot spot to the west and a cooler plateau to the east can be seen in the two maps (Figs. 13b and 14c), which shows a similar relationship of temperatures as between the MSX and HIRES $(T(12 / 25))$ data.

\section{Conclusions}

A detailed radio and infrared study of an embedded young stellar cluster associated with the Galactic star forming region S 201 is presented. Colour-colour and colour-magnitude diagrams have been constructed to identify young stellar objects and estimate their spectral types. High sensitivity and high angular resolution radio continuum maps based on the GMRT observations at 610 and $1280 \mathrm{MHz}$ have been generated. These radio maps show interesting morphological details, including an arc-shaped structure highlighting the interaction between the HII region and the adjacent molecular cloud. Three luminous infrared sources have been identified located within the radio nebulosity. The spatial distribution of temperature and optical depth of the interstellar dust component in the S 201 region has been presented based on the mid- and far-infrared measurements from the MSX and IRAS (HIRES) missions.

We have detected a compact embedded star cluster located within the ionized nebula responsible for the radio continuum emission. The cluster has a richness similar to the clusters surrounding early type Herbig Be stars; the most luminous member is consistent with an O6-O8 zero age main sequence star. Our analysis confirms that this single star is responsible for most of the Lyman continuum emission required to sustain the ionized radio nebula, with all the other cluster members contributing at most $10 \%$ of the ionizing photons. This explains the success of the model of Felli et al. (1987) (which assumed a single massive star for excitation) in explaining the radio continuum morphology of S 201. Our observations also confirm the position of this massive star as well as the location of the molecular cloud being eroded by the ionizing radiation. The PDR at the interface between the ionized gas and the molecular cloud is traced to the west of the bright arc-shaped 

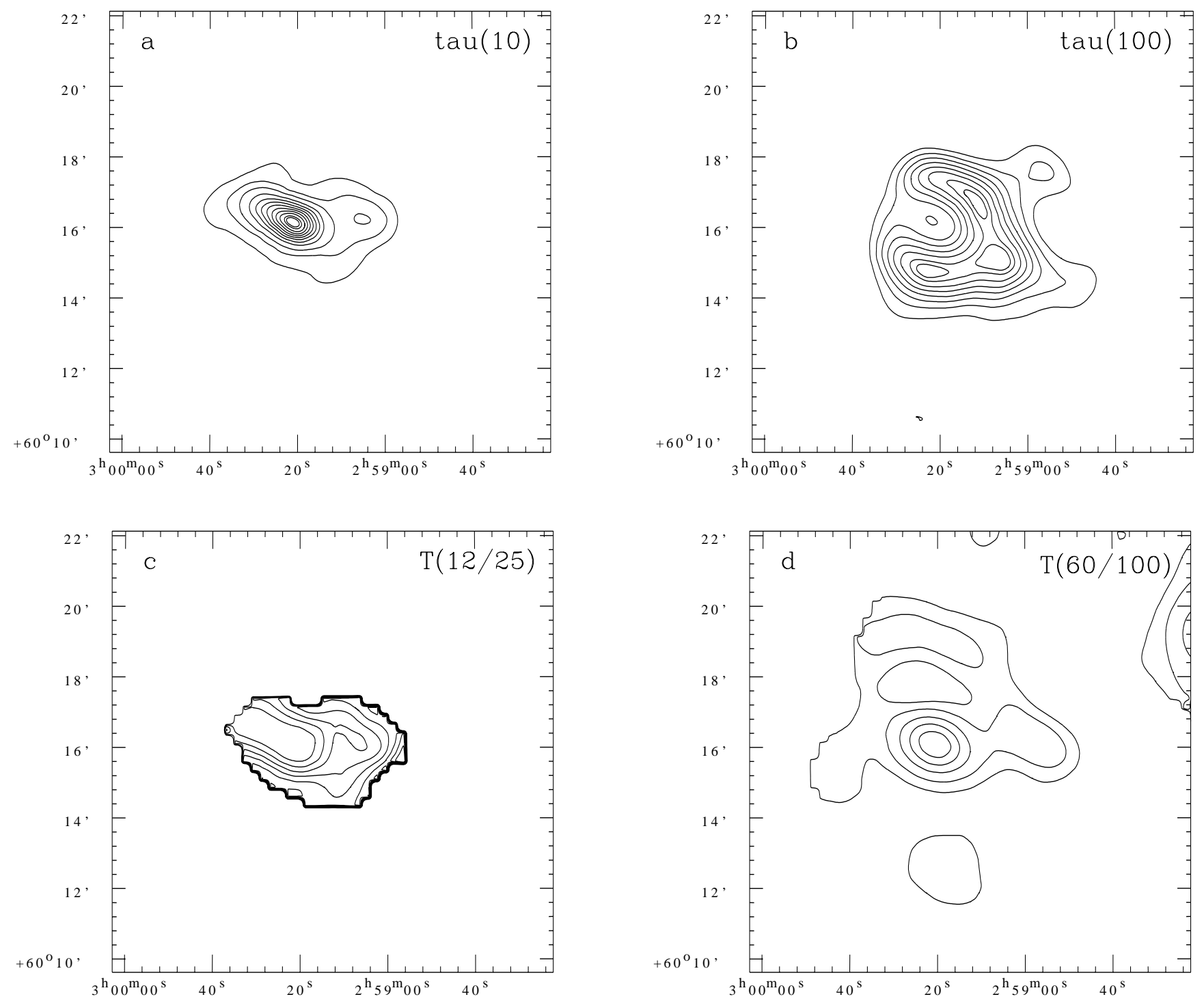

Fig. 14. a) The dust optical depth $\left(\tau_{10}\right)$ distribution from the HIRES 12 and $25 \mu \mathrm{m}$ maps, scaled to $10 \mu \mathrm{m}$ by $\lambda^{-1}$ emissivity law. The contours represent $95,90,80,70,60,50,40,30,20,10,5$ and $2 \%$ of the global peak value of $6.63 \times 10^{-5}$. b) The dust optical depth $\left(\tau_{100}\right)$ distribution from the HIRES 60 and $100 \mu \mathrm{m}$ maps for the region around S 201 assuming a dust emissivity law of $\epsilon_{\lambda} \propto \lambda^{-1}$. The contours represent 95, 90, $80,70,60,50,40$ and $30 \%$ of the global peak value of $3.05 \times 10^{-3}$.c $)$ The dust temperature $(T(12 / 25))$ distribution and the contours are drawn from 140,145 to $215 \mathrm{~K}$ in step of $10 \mathrm{~K}$ (from left to right in the figure). The peak value in this map is $230 \mathrm{~K}$ and occurs at the extreme right position (RA $=02: 58: 56.4, \mathrm{dec}=+60: 15: 38)$ of the map. $\mathbf{d}$ ) The dust temperature $(T(60 / 100)$ ) distribution and the contours correspond to the temperatures 55, 50, 45, 40 and $37 \mathrm{~K}$. The abscissa and the ordinates are in B1950.0 epoch.

feature in radio continuum and $\mathrm{Br} \gamma$, by the diffuse $\mathrm{H}_{2}$ emission detected in our narrow-band near-infrared images.

Acknowledgements. We thank the staff of the GMRT that made the radio observations possible. The GMRT is run by the National Centre for Radio Astrophysics of the Tata Institute of Fundamental Research. It is a pleasure to thank the Arcetri and TNG technical staff and the TNG operators for their assistance during the NICS observing runs. We also thank the TIRGO team, especially Filippo Mannucci, for helpful scheduling and service observing at the Gornergrat.

This research made use of data products from the Midcourse Space Experiment, the processing of which was funded by the Ballistic Missile Defense Organization with additional support from from NASA Office of Space Science. This research has also made use of the NASA/IPAC Infrared Science Archive, which is operated by the Jet Propulsion Laboratory, California Institute of Technology, under contract with National Aeronautics and Space Administration.

D.K.O. was supported by the JSPS (Japan) through a fellowship during which some part of this work was done. We thank Francesco Palla for providing us with their PMS grids.

\section{References}

Aumann, H. H., Fowler, J. W., \& Melnyk, M. 1990, AJ, 99, 1674 Bachiller, R., Fuente, A., \& Kumar, M. S. N. 2002, A\&A, 381, 168 Baffa, C., Comoretto, G., Gennari, S., et al. 2001, A\&A, 378, 722 Blair, G. N., Dinger, A. C., \& Dickinson, D. F. 1980, AJ, 85, 161 Carpenter, J. M., Snell, R. L., Schloerb, F. P., et al. 1993, ApJ, 407, 657

Felli, M., Hjellming, R. M., \& Cesaroni, R. 1987, A\&A, 182, 313

Fich, M. 1993, ApJS, 86, 475 
Ghosh, S. K., Verma, R. P., Rengarajan, T. N., et al. 1993, ApJS, 86, 401

Ghosh, S. K., \& Ojha, D. K. 2002, A\&A, 388, 326

Hunt, L. K., Mannucci, F., Testi, L., et al. 1998, AJ, 115, 2594

Kleinmann, S. G., Joyce, R. R., Sargent, D. G., et al. 1979, ApJ, 227, 126

Koornneef, J. 1983, A\&A, 128, 84

Mampaso, A., Vilches, J. M., Pismis, P., et al. 1987, RMxAA, 14, 474

Mampaso, A., Vilches, J. M., Riera, A., et al. 1989, A\&A, 220, 235

Martin, R. N., \& Barrett, A. H. 1978, ApJS, 36, 1

Meyer, M. R., Calvet, N., \& Hillenbrand, L. 1997, AJ, 114, 288

Omar, A., Chengalur, J. N., \& Roshi, D. A. 2002, A\&A, 395, 227

Palla, F., \& Stahler, S. 1999, ApJ, 525, 772
Panagia, N. 1973, AJ, 78, 929

Price, S. D., Egan, M. P., Carey, S. J., et al. 2001, AJ, 121, 2819

Rieke, G. H., \& Lebofsky, M. J. 1985, ApJ, 288, 618

Stetson, P. B. 1987, PASP, 99, 191

Swarup, G., Ananthakrishnan, S., Kapahi, V. K., et al. 1991, Curr. Sci., 60, 95

Testi, L., Palla, F., \& Natta, A. 1998, A\&AS, 133, 81

Testi, L., Palla, F., \& Natta, A. 1999, A\&A, 342, 515

Thronson, H. A., Smith, H. A., Glaccum, W., et al. 1984, MNRAS, 207, 659

Walmsley, C. M., Natta, A., Oliva, E., \& Testi, L. 2000, A\&A, 364, 301

Zinchenko, I., Henning, Th., \& Schreyer, K. 1997, A\&AS, 124, 385 